CONTRIBUCIÓN DEL $3^{\text {ER }}$ CONGRESO LATINOAMERICANO DE MACROINVERTEBRADOS DE AGUA DULCE: BIODIVERSIDAD Y ECOLOGÍA FUNCIONAL EN EL NEOTRÓPICO

\title{
ESTRUCTURA TRÓFICA DE LOS INVERTEBRADOS ACUÁTICOS ASOCIADOS A Egeria densa (PLANCH. 1849) EN EL LAGO DE TOTA (BOYACÁ-COLOMBIA)
}

\author{
TROPHIC STRUCTURE OF AQUATIC INVERTEBRATES ASSOCIATED WITH Egeria densa \\ (PLANCH. 1849) IN TOTA LAKE (BOYACÁ-COLOMBIA)
}

Adriana Pedroza-Ramos, Pedro Caraballo y Nelson Aranguren-Riaño

\begin{abstract}
RESUMEN
Una forma fundamental y efectiva de visualizar los patrones y propiedades dinámicas de una comunidad, es a través de las redes tróficas, las cuales son entendidas como el mapa de las interrelaciones entre la estructura de las comunidades con los procesos que ocurren dentro del ecosistema. En este estudio se describe una red trófica acuática, conformada por invertebrados acuáticos, detritus y perifiton, asociada con la planta Egeria densa. Se realizaron muestreos en periodos de transición y lluvias, en cuatro estaciones en la zona litoral del Lago de Tota. En cada sitio se recolectaron tres muestras de $300 \mathrm{~g}$ de material vegetal. Se midieron in situ los siguientes parámetros: conductividad eléctrica, $\mathrm{pH}$, oxígeno disuelto y temperatura. Por medio de información bibliográfica y en algunos casos mediante observación, se asignaron los hábitos alimenticios. Finalmente, se desarrolló un modelo de trama trófica tipo estático. Se registraron 112866 individuos distribuidos en 26 taxones que fueron clasificados según sus hábitos alimenticios en: dos trofoespecies tope, 16 intermedias y dos basales. Esta red trófica presentó una conectancia media de 0,13 y una densidad de uniones de 2,95. En conjunto, estos resultados muestran a E. densa como generadora de detritus y sustrato de perifiton, consideradas en el modelo como trofoespecies basales que sustentan la comunidad. Además de la presencia de 10 trofoespecies consumidoras primarias que generan alta redundancia ecológica y a la vez garantizan el flujo de recursos hacia niveles tróficos superiores.
\end{abstract}

PALABRAS CLAVE: lagos de alta montaña, trópico, Red trófica, Trofoespecies

\begin{abstract}
A fundamental and effective way to view the dynamic patterns and properties of a community is through the trophic network, understood as a map of the interrelationships between the structure of an ecological community and the processes that occur within the ecosystem. In this study, we describe an aquatic food web composed by aquatic invertebrates, detritus and periphyton associated with the plant Egeria densa. Samples were collected during the transitional and rainy season at four stations in the littoral zone of Tota Lake. Three samples (300 g each) of plant material at every point were randomly collected. Electrical conductivity, $\mathrm{pH}$, dissolved oxygen and temperature were measured in situ. The eating habits were assigned by means of bibliographic information and in some cases observation. Finally, we developed a static type model that included 112866 individuals distributed among 26 taxon, which were classified according to their eating habits in: two top trophic species, 16 intermediate and two basal species. We found that this trophic network represents a connectance of 0.13 and density links of 2.95. The model also revealed that $E$. densa is a basal trophic species. Together, these results demonstrate that $E$. densa is an important generator of detritus and periphyton substrate, supporting other trophic levels in the community. Moreover, the presence of ten trophic species of primary consumers generates high ecological redundancy that at the same time guarantees the flow of resources toward higher trophic levels.
\end{abstract}

KEY WORDS: food web, high mountain lakes, tropic, trophic species 


\section{INTRODUCCIÓN}

La zona litoral de los ecosistemas leníticos continentales ofrece mayor cantidad de hábitats en comparación con la zona limnética, de ahí que la riqueza de especies en dicha zona sea también más alta (Rico-Sánchez et al., 2014). En general, los estudios sobre cadenas tróficas en los lagos, son realizados con base en muestreos en la zona limnética, hecho que limita el análisis de las interacciones que existen entre los diferentes compartimientos de estos ecosistemas. Claramente la zona que ha concentrado la atención en los estudios ecológicos ha sido la limnética, dejando de lado las zona riparía, litoral y bentónica, a menudo concebidas únicamente como fuente o sumidero de nutrientes y no como parte integral de las cadenas tróficas (Schindler y Scheuerell, 2002; Vadeboncoeur et al., 2002). Por tales razones, se encuentra necesario incluir en nuevos estudios muestreos en varias zonas de los ecosistemas leníticos y de manera especial, abordar las zonas litorales con influencia de macrofitos acuáticos, ya que muchas de las interacciones bióticas que son críticas para la integridad de estos ecosistemas ocurren en estas zona (Caraballo et al., 2014).

La planta Egeria densa es un macrófito acuático sumergido y enraizado, asociado con múltiples problemas ambientales, por las cuales es considerado como maleza en muchas regiones (Yarrow et al., 2009), se caracteriza por su alta tasa de colonización y de producción, alcanza aproximadamente $522 \mathrm{~g} / \mathrm{m}^{2}$ como resultado de la combinación entre una baja tasa de senescencia y su continuo crecimiento (Carrillo et al., 2006). Estas características le permiten consolidar densos cinturones en la región litoral, como los observados por Hernani y Ramírez (2002), por tanto, tiene un papel estructurador en estos ecosistemas, definiendo una compleja estructura subacuática, modelando y generando efectos físicos, metabólicos, biológicos y sobre las interacciones bióticas que definen la estructura de la comunidad (Yarrow et al., 2009). Ahora bien, a pesar de que existen pocos registros de herbivoría directa sobre esta planta (Walsh et al., 2013), su uso como hábitat de algas epifitas, invertebrados y pequeños peces está bien documentado (Wetzel y Allen, 1972; Howard-Williams y Lenton, 1975; Pelicice et al., 2005; Croteau et al., 2005; Giorgi y Feijoo, 2010) así como su aporte al detritos (Frauendorf et al., 2013). El detrito es considerado como un recurso dinámico y heterogéneo, que sirve de hábitat, refugio y sitio de reproducción, convirtiéndose en un componente integral de las llamadas actividades de ingeniería de los ecosistemas y constituye un recurso básico para organismos detritívoros y descomponedores (Moore et al., 2004). Lo anterior muestra la importancia de E. densa en la estructuración de las comunidades presentes en los ecosistemas acuáticos, en especial de las relaciones tróficas que allí se desarrollan.

Con relación a la diversidad de animales que habitan la zona litoral de los sistemas lénticos, se sabe, que en su mayoría, está representada por la comunidad de invertebrados, tanto los que viven en íntima asociación con la vegetación (gasterópodos, insectos, crustáceos), así como los organismos de vida libre (zooplancton) (Thomaz y Cunha, 2010; Esteves, 2011). En la actualidad, se reconoce que la función de estos organismos, puede entenderse mejor mediante la descripción de sus rasgos funcionales, actividades, y uso de recursos (Ramírez y Gutiérrez-Fonseca, 2014; Salgado-Negret, 2015).

El uso de las redes tróficas, es entendido como el mapa de las interrelaciones entre la estructura de una comunidad ecológica, con su estabilidad y con los procesos que están ocurriendo dentro de un ecosistema (De Ruiter et al., 2005), tiene como ventaja que evidencia relaciones básicas como la herbívora y la depredación e incluso casos de extinción secundaria, que se presentan cuando una especie fuertemente conectada es removida del sistema (Dunne et al., 2002). Sin embargo, tiene como desventaja que trivializa relaciones complejas (como consecuencia de la agrupación de organismos diferentes), que pueden asumirse como estáticas cuando en la realidad son profundamente dinámicas (Caraballo, 2009). Desde el punto de vista de la estructura o topología, las redes tróficas están constituidas por nodos, eslabones y niveles tróficos (Pimm et al., 1991). En ese sentido, la riqueza de una red trófica no solo es función de la riqueza biológica de una comunidad, sino también del nivel de resolución adoptado por los investigadores para la definición de las trofoespecies (Giacomini y Petrere, 2010), entendidas como un agregado de especies que comparten depredador y/o presa en una red trófica (Pimm et al., 1991).

En general, en las redes tróficas se encuentran tres tipos de especies o trofoespecies (Pimm et al., 1991): (a) trofoespecies basales, que sólo son presas pues no depredan, allí están los autótrofos y el detrito (considerado como una trofoespecie funcional); (b) trofoespecies intermedias, tienen al menos un depredador y al menos una presa; (c) trofoespecies tope, no tienen depredadores en el ecosistema. Así, con el propósito de establecer el nivel de estabilidad de una 
red trófica, se puede considerar la conectancia, cuyos valores oscilan entre 0,03 y 0,3 , con valores medios entre 0,1 y 0,15 (Dunne y Williams, 2009).

Estos ecosistemas constituidos por un conjunto de elementos y conjuntos de relaciones entre estos elementos, se puede traducir en un lenguaje lógico y matemático (Margalef, 1983), elementos base para la construcción de modelos. En las redes tróficas se distinguen al menos dos categorías: el modelo de trama trófica estática y el modelo trofodinámico (AndradeLópez, 2012). La opción de usar información cualitativa para la construcción de modelos es exigente y tienen aplicabilidad en la comprensión de sistemas de alta complejidad (Nash y Sutcliffe, 1970).

En la medida en que los recursos alimenticios y los predadores están entre los principales factores limitantes del crecimiento poblacional de cualquier especie, las interacciones tróficas son componentes esenciales para entender la dinámica de las poblaciones y consecuentemente de los patrones emergentes de coexistencia y diversidad en los ecosistemas (Giacomini, 2007). Es en este contexto se presenta esta investigación, cuyo objetivo es determinar la estructura trófica de los invertebrados acuáticos asociados a $E$. densa en la zona litoral del lago de Tota, mediante la construcción de una red trófica representada en un modelo estático.

\section{MATERIALES Y MÉTODOS}

\section{Área de Estudio}

El lago de Tota está ubicado entre los municipios de Aquitania, Tota y Cuítiva $\left(5^{\circ} 28^{\prime}\right.$ y $5^{\circ} 39^{\prime} \mathrm{N}, 72^{\circ} 51^{\prime}$ y $73^{\circ} 0^{\prime} \mathrm{W}$ ), que hacen parte de la cordillera Oriental del departamento de Boyacá, a una altitud de $3015 \mathrm{~m}$. Cuenta con una área de $60 \mathrm{~km}^{2}$, un volumen de 1920 millones de $\mathrm{m}^{3}$, una profundidad máxima de $60 \mathrm{~m}$ y una temperatura media durante el día de $11^{\circ} \mathrm{C}$ (Cordero et al., 2005). Durante años este sistema se ha visto influenciado por diferentes actividades antropogénicas que se describen a continuación:

1. Agricultura intensiva: Se desarrolla en la cuenca del lago, el cultivo más representativo es el de cebolla junca (Allium fistulosum), con una producción de 131451 toneladas de cebolla por año y un rendimiento de 34,53 toneladas por hectárea, convirtiéndose en una de las zonas con mayor producción del país. Esta actividad genera, por erosión, escorrentía y/o lixiviación, la incorporación al lago de agroquímicos, abonos orgánicos y plaguicidas con altos contenidos de malatión, difenoconazol, tebuconazol, entre otros (Mojica y Guerrero, 2013).

2. Descarga directa de aguas residuales, en especial las del municipio de Aquitania, en donde la planta de tratamiento no funciona adecuadamente y aproximadamente el $55 \%$ de las viviendas vierten las aguas residuales a los cultivos, potreros o caños (Ricaurte, 2005).

Estas actividades son fuentes potenciales de contaminación para el lago, el cual muestra signos de eutrofización, evidenciados por altos valores de clorofila-a y aumento de nutrientes (fósforo y nitrógeno), con mayor grado de concentración en la sub-cuenca Lago Chico, principalmente por los aportes del río La Mugre y Tobal (Cardozo et al., 2005; Corpoboyaca, 2015).

\section{Fase de Campo}

Teniendo en cuenta la presencia de E. densa y las categorías de calidad ambiental (baja, aceptable e intermedia), propuestas por Gil y Aranguren (2013) y Gil-Padilla et al. (2016), para la zona litoral del Lago, se seleccionaron cuatro estaciones de muestreo relacionadas en la figura 1, que corresponden (a) Pispesca: presenta una categoría aceptable con altos valores de oxígeno, bajos de conductividad y bajas densidades de macroinvertebrados; (b) La Custodia: con una categoría intermedia; (c) Llano de Alarcón: con una categoría baja, que corresponde a bajos valores de oxígeno, altos de conductividad y altas densidades de macroinvertebrados; (d) Playa Blanca se incluyó como una estación adicional.

La recolección de muestras se realizó en dos periodos climáticos (transición y lluvias). En cada estación fueron extraídas tres muestras con un peso fresco de $300 \mathrm{~g} \mathrm{c} / \mathrm{u}$, esto se hizo empleando una red de mano con poro de malla de $3 \mathrm{~mm}$. Las muestras se almacenaron en bolsas de cierre hermético y se fijaron en alcohol al 70 \% (Wetzel y Likens, 2000).

Simultáneamente con el muestreo biológico se midieron in situ las variables recomendados por Ruíz (2002) para el estudio de ambientes acuaticos: temperatura del agua, oxígeno disuelto, porcentaje de saturación de oxígeno (oxímetro YSI 55-12FT), conductividad eléctrica (conductímetro YSI 85) y el pH (pH-metro Schott portátil). 


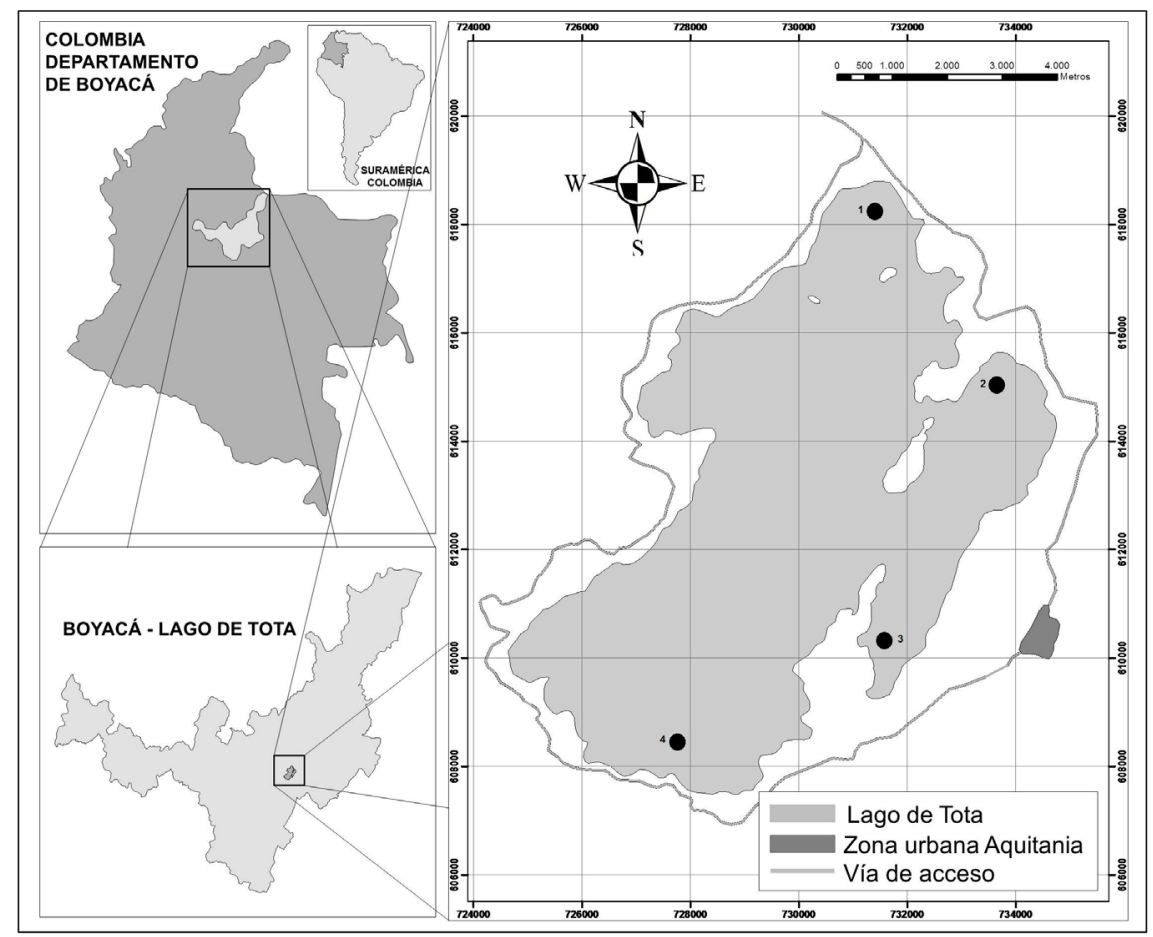

Figura 1. Mapa del Lago de Tota y ubicación de las cuatro estaciones de muestreo (1-llano Alarcón, 2-Pispesca, 3-La Custodia, 4-Playa Blanca).

\section{Fase de Laboratorio}

Las muestras de $E$. densa se lavaron con agua sobre un tamiz con poro de malla de $250 \mu \mathrm{m}$, posteriormente los invertebrados obtenidos se depositaron en frascos con alcohol al $70 \%$, éstos se identificaron al nivel de resolución más específico posible con base en las claves de McCafferty y Provonsha (1981); Pennak (1989); Brinkhurst y Marchese (1991); Novelo y Gonzalez (1991); Epler (1992); Roldán (1996); ElmoorLoureiro (1997); FlöBner (2000); Ruiz-Moreno et al. (2000); Dumont y Negrea (2002); Gaviria y Aranguren (2003); Domínguez y Fernández (2009); Springer et al. (2010); además se contó con la asesoría taxonómica por parte de especialistas para los grupos Naididae, Ostracoda, Arachnida, Trichoptera, Hemiptera, Odonata y Chironomidae.

Los hábitos alimenticios se establecieron por medio de revisión de literatura y se corroboró la información por medio de observaciones personales en laboratorio, a través del cuidado y observación del ciclo de vida de los siguientes taxones: Amphipoda, Odonata, Coleoptera,
Trichoptera, Diptera. Se recolectaron organismos de diferentes estadios y se trasladaron a los laboratorios de la UPTC, en donde se separaron y depositaron en vasos de poliestireno, que fueron cubiertos con papel parafilm para evitar la pérdida de los organismos, en estos recipientes se criaron y alimentaron con organismo recolectados en el mismo sistema.

\section{Tratamiento de Datos}

La información ambiental (variables físicas y químicas), se interpretó mediante estadística descriptiva, utilizando la media aritmética y el coeficiente de variación (Guisande et al., 2006).

La red trófica se estableció mediante un modelo estático o topológico, el cual se basa en una matriz binaria (presencia / ausencia de conexiones), donde se registran cuales taxones dentro del sistema se alimentan de otros y las interacciones tróficas pueden estar presentes 0 ausentes entre cada par de taxones (Andrade-López, 2012). Se empleó el programa de libre acceso Gephi 0,9,1 (Bastian et al., 2009), software altamente utilizado 
para el diseño de grafos, por sus bondades visuales con el manejo de la informacion, estructuras, formas y patrones.

Para la interpretación de este modelo se midieron los siguientes atributos:

Conectancia: Hace referencia a la proporción de los posibles vínculos en una red alimentaria. Para este caso se utilizó la conectancia dirigida $\left(\mathrm{C}=\mathrm{L} / \mathrm{S}^{2}\right)$, en la que se consideran todas las posibles conexiones y donde $L$ corresponde al número de conexiones observadas y $S$ al número de especies tróficas (Pimm et al., 1991).

Densidad: Con esta opción se mide el número de conexiones (entradas o salidas) desde y hacia una especie (L / S), donde L corresponde al número de conexiones presentes y $S$ el número de especies tróficas (Dunne, 2009).

\section{RESULTADOS}

Las variables físicas y químicas se presentan de manera informativa, éstas no mostraron variaciones importantes entre las estaciones y los muestreos.
Las estaciones que presentaron mayor coeficiente de variación fueron: Llano de Alarcón y La Custodia, con temperaturas altas y bajos valores de oxígeno, pero en general, se detectaron $\mathrm{pH}$ alcalinos, con saturación de oxígeno disuelto por encima del $50 \%$ y conductividades con valores que oscilaron entre 80,7 y $87,4 \mu \mathrm{S} . \mathrm{cm}^{-1}$ (Tabla 1 ).

Se recolectaron 112866 organismos, pertenecientes a 16 órdenes, 23 familias y 24 géneros (Tabla 2). El género más abundante en el total de estaciones fue Dicrotendipes sp. (Chironomidae), el cual alcanzó valores de hasta 8433 individuos por estación que representa el $65 \%$ del total de la abundancia de la comunidad, mostrando clara dominancia sobre los demás taxones. Otros taxones que presentaron altas abundancias fueron los cladóceros (Eurycercidae y Chydoridae) con valores de hasta 4775 individuos que representa el $30 \%$ y los ácaros (Hydrozetes) con hasta 2473 que representa el $19 \%$ de la abundancia. Por otro lado el grupo de los gasterópodos (Planorbis y Ferrisia), mostró bajas abundancias, con valores que oscilaron entre 0,04 y $2,82 \%$. En general la composición de invertebrados no varió entre estaciones y muestreos, pero la proporción de sus abundancias si tuvo variaciones.

Tabla 1. Información de las variables físicas y químicas medidas in situ en el Lago de Tota, durante el periodo de estudio.

\begin{tabular}{|c|c|c|c|c|c|c|c|c|c|c|}
\hline \multirow{2}{*}{ Estación } & \multicolumn{2}{|c|}{$\begin{array}{l}\text { Temperatura } \\
\quad\left({ }^{\circ} \mathrm{C}\right)\end{array}$} & \multicolumn{2}{|c|}{$\begin{array}{l}\text { Conductividad } \\
\left(\mu \mathrm{S} . \mathrm{cm}^{-1}\right)\end{array}$} & \multicolumn{2}{|c|}{$\begin{array}{c}\mathrm{pH} \\
\text { (Unidades) }\end{array}$} & \multicolumn{2}{|c|}{$\begin{array}{c}\text { O.D } \\
\left(\mathrm{mg} . \mathrm{l}^{-1}\right)\end{array}$} & \multicolumn{2}{|c|}{$\begin{array}{l}\% \text { SAT } \\
(\% 02)\end{array}$} \\
\hline & $\bar{X}$ & CV \% & $\bar{X}$ & CV \% & $\bar{X}$ & CV \% & $\bar{X}$ & CV \% & $\bar{X}$ & CV \% \\
\hline Llano Alarcón & 17,08 & 6,36 & 83,85 & 1,71 & 8,67 & 5,60 & 6,54 & 22,03 & 66,68 & 26,20 \\
\hline Pispesca & 17,18 & 5,19 & 87,3 & 1,05 & 8,60 & 5,37 & 6,59 & 8,07 & 68,95 & 14,27 \\
\hline La Custodia & 18,65 & 26,63 & 82,8 & 2,47 & 8,01 & 2,89 & 5,48 & 19,09 & 55,11 & 20,67 \\
\hline Playa Blanca & 16,73 & 4,15 & 84,46 & 1,78 & 8,29 & 2,83 & 5,71 & 17,30 & 58,36 & 19,73 \\
\hline
\end{tabular}

Definidos los taxones (Tabla 2) y las preferencias alimenticias de éstos (Tabla 3 ), se agruparon los organismos en 20 trofoespecies que se representaron en un modelo de red trófica (Figura 2). Una vez construido el modelo de red trófica, se determinaron sus atributos topológicos, donde se muestra que el 10 \% corresponde a trofoespecies tope (Coleóptero: Gyrinus y Tricladida:
Girardia), el $10 \%$ a trofoespecies basales (Perifiton y Detritus), y el $80 \%$ restante corresponde a trofoespecies intermedias de las cuales, el $10 \%$ corresponden a trofoespecies caníbales (Odonatos: Ischnura hastata, Coryphaeschna y el Hemiptero: Neosigara). Los cálculos muestran una conectancia dirigida de 0,13 y una densidad de conexiones de 2,95. 
Tabla 2. Listado taxonómico de invertebrados acuáticos asociados a E. densa, con sus respectivas abundancias relativas (\%), para las dos épocas estudiadas. Orden sistemático según: Brusca y Brusca (2003).

\begin{tabular}{|c|c|c|c|c|c|c|c|c|}
\hline \multirow{2}{*}{ Taxones Reportados } & \multicolumn{2}{|c|}{ Llano de Alarcón } & \multicolumn{2}{|c|}{ Pispesca } & \multicolumn{2}{|c|}{ La Custodia } & \multicolumn{2}{|c|}{ Playa Blanca } \\
\hline & 1 & 2 & 1 & 2 & 1 & 2 & 1 & 2 \\
\hline Girardia sp. Ball, 1974 & 0,3 & 0,97 & 0,15 & 1,51 & 0,34 & 0,06 & 0,76 & 0,1 \\
\hline Nais sp. Müller, 1774 & 0,56 & 2,12 & 0 & 4,85 & 4,97 & 14,63 & 2,92 & 5,74 \\
\hline $\begin{array}{l}\text { Helobdella sp. Blanchard, } \\
1896\end{array}$ & 0 & 0,01 & 0,12 & 0,07 & 0 & 0 & 0,03 & 0 \\
\hline $\begin{array}{l}\text { Chydoridae } \\
\text { (Grabtoleberis sp. Sars, } \\
\text { 1862, Alonella sp. Sars, } \\
\text { 1863) }\end{array}$ & 8,89 & 16,02 & 1,39 & 10,52 & 17,87 & 1,4 & 12,12 & 6,27 \\
\hline $\begin{array}{l}\text { Simocephalus sp. } \\
\text { Schoedler, } 1858\end{array}$ & 7,98 & 0,01 & 5,08 & 5,94 & 2,33 & 2,49 & 1,98 & 3,62 \\
\hline $\begin{array}{l}\text { Eurycercus norandinus. } \\
\text { Aranguren, Gaviria y } \\
\text { Monrroy, } 2002\end{array}$ & 7,38 & 0,03 & 2,15 & 7,02 & 0,08 & 0,32 & 12,53 & 30,49 \\
\hline $\begin{array}{l}\text { Macrothrix sp. Baird, } \\
1843\end{array}$ & 11,02 & 0,76 & 6,38 & 4,04 & 0,41 & 0,13 & 0,4 & 1,16 \\
\hline $\begin{array}{l}\text { Hyalella paramoensis. } \\
\text { Andres, } 1988\end{array}$ & 6,11 & 1,71 & 0,02 & 0,58 & 0,95 & 0 & 8,08 & 3,56 \\
\hline $\begin{array}{l}\text { Macrocyclops albidus. } \\
\text { Jurine, } 1820\end{array}$ & 1,52 & 0,78 & 0,63 & 1,12 & 5,55 & 1,21 & 0,64 & 0,26 \\
\hline $\begin{array}{l}\text { Cypridopsis vidua. } \\
\text { Müller, } 1776\end{array}$ & 2,51 & 7,55 & 7,34 & 6,57 & 5,33 & 1,15 & 7,46 & 5,32 \\
\hline $\begin{array}{l}\text { Hydrozetes cf. } \\
\text { dimorphus. Hammer, } \\
1962\end{array}$ & 6,29 & 15,57 & 18,89 & 14,64 & 22,54 & 5,49 & 5,68 & 3,92 \\
\hline $\begin{array}{l}\text { Trimalaconothrus cf. } \\
\text { novus. Sellnick, } 1921\end{array}$ & 1,95 & 8,8 & 0,12 & 0,61 & 12,28 & 3,83 & 2,91 & 7,66 \\
\hline $\begin{array}{l}\text { Halacaridae. Murray, } \\
1877\end{array}$ & 0 & 0,01 & 0 & 0,07 & 0,34 & 0,06 & 0,18 & 0,26 \\
\hline $\begin{array}{l}\text { Odonata: Coryphaeschna } \\
\text { sp. Williamson, 1903; } \\
\text { Ischnura hastata. Say, } \\
1839\end{array}$ & 0,21 & 0,06 & 0,2 & 0,11 & 0,43 & 0,25 & 0,32 & 0,1 \\
\hline $\begin{array}{l}\text { Neosigara sp. Lundblad, } \\
1928\end{array}$ & 0,13 & 0 & 1,96 & 1,28 & 0,05 & 0 & 0 & 0 \\
\hline $\begin{array}{l}\text { Gyrinus sp. Geoffroy, } \\
1762\end{array}$ & 0,13 & 0 & 0 & 0,15 & 0 & 0 & 0,09 & 0 \\
\hline
\end{tabular}


Continuación Tabla 2.

\begin{tabular}{|c|c|c|c|c|c|c|c|c|}
\hline \multirow{2}{*}{ Taxones Reportados } & \multicolumn{2}{|c|}{ Llano de Alarcón } & \multicolumn{2}{|c|}{ Pispesca } & \multicolumn{2}{|c|}{ La Custodia } & \multicolumn{2}{|c|}{ Playa Blanca } \\
\hline & 1 & 2 & 1 & 2 & 1 & 2 & 1 & 2 \\
\hline $\begin{array}{l}\text { Oxyethira sp. Eaton, } \\
1873\end{array}$ & 0,17 & 2,5 & 0,78 & 0,42 & 1,88 & 0,32 & 6,69 & 4,89 \\
\hline $\begin{array}{l}\text { Dicrotendipes sp. Kieffer, } \\
1913\end{array}$ & 41,18 & 40,63 & 50,41 & 35,91 & 21,34 & 64,67 & 34 & 25,38 \\
\hline $\begin{array}{l}\text { Ablabesmyia sp. } \\
\text { Johannsen, } 1905\end{array}$ & 0,52 & 2,21 & 1,83 & 3,61 & 0,5 & 0,38 & 0,24 & 0,26 \\
\hline $\begin{array}{l}\text { Hemerodromia sp. } \\
\text { Meigen, } 1822\end{array}$ & 0 & 0,04 & 0,02 & 0 & 0 & 0 & 0,03 & 0 \\
\hline $\begin{array}{l}\text { Chelifera sp. Macquart, } \\
1823\end{array}$ & 0,13 & 0 & 0 & 0 & 0,05 & 0,06 & 0,03 & 0 \\
\hline $\begin{array}{l}\text { Physella sp. Haldeman, } \\
1842\end{array}$ & 2,82 & 0,04 & 1,39 & 0,23 & 1,04 & 0,06 & 0,43 & 0,37 \\
\hline $\begin{array}{l}\text { Planorbis sp. Müller, } \\
1774\end{array}$ & 0 & 0 & 0,02 & 0 & 0 & 0 & 0,18 & 0 \\
\hline Ferrissia sp. Walker, 1903 & 0,13 & 0,14 & 0 & 0 & 1,63 & 3,45 & 2,21 & 0,53 \\
\hline
\end{tabular}

Tabla 3. Preferencias alimenticias de los invertebrados acuáticos asociados a E. densa registrados durante el periodo de estudio en la zona litoral del Lago de Tota.

\begin{tabular}{lll}
\hline Predador & Presa & Referencia \\
\hline Girardia & $\begin{array}{l}\text { Quironómidos, tubifícidos, Gammarus, } \\
\text { cladóceros. }\end{array}$ & Pickavance (1971) \\
Nais & Algas, detritus & Smith y Kaster (1986) \\
Helobdella & Quironómidos, oligoquetos, gasterópodos. & Ironmonger y Young (1980) \\
$\begin{array}{l}\text { Alonella, Eurycercus } \\
\text { Grabtoleberis }\end{array}$ norandinus, & Detritus y algas & Fryer (1968) \\
Macrothrix, Simocephalus & & \\
Hyalella paramoensis & Diatomeas y detritos & Observación personal \\
Harpacticoida & Microalgas, bacterias y detritos & Limén al. (2005) \\
& Culícidos, protozoos, rotíferos, & Berghe y Bergmans (1981) \\
Macrocyclops abidus & quironómidos, larvas de anfibio y & Marten (1990) \\
& mosquitos. & Suárez et al. (2005) \\
Cypridopsis vidua & Perifiton & Margalef (1983) \\
Hydrozetes Trimalaconothrus, & Algas unicelulares y filamentosas, hongos y & Roca et al. (1993) \\
& material vegetal en descomposición. & Thorp y Covich (2010) \\
\hline
\end{tabular}


Continuación Tabla 3.

\begin{tabular}{|c|c|c|}
\hline Predador & Presa & Referencia \\
\hline Ischnura hastata, Coryphaeschna & $\begin{array}{l}\text { Quironómidos, cladóceros, moluscos, } \\
\text { oligoquetos, gasterópodos, ostrácodos, } \\
\text { tricópteros y otros macroinvertebrados } \\
\text { acuáticos. }\end{array}$ & $\begin{array}{l}\text { Observación personal } \\
\text { Pritchard (1964) } \\
\text { Merrit et al. (2008) }\end{array}$ \\
\hline Corixidae & $\begin{array}{l}\text { Algas filamentosas, detritos, pequeños } \\
\text { crustáceos, quironómidos, larvas de } \\
\text { mosquito, y oligoquetos. }\end{array}$ & $\begin{array}{l}\text { Martin (1969) } \\
\text { Klecka (2014) }\end{array}$ \\
\hline Gyrinus & $\begin{array}{l}\text { Animales de cuerpos blandos, oligoquetos, } \\
\text { larvas de quironómidos y ninfas de } \\
\text { odonatos. }\end{array}$ & $\begin{array}{l}\text { Observación personal } \\
\text { Oscoz et al. (2011) }\end{array}$ \\
\hline Oxyethira & Algas & $\begin{array}{l}\text { Observación personal } \\
\text { Keiper et al. (1898) }\end{array}$ \\
\hline Dicrotendipes & Detritos & $\begin{array}{l}\text { Observacion personal } \\
\text { Merrit et al. (2008) }\end{array}$ \\
\hline Ablabesmyia & $\begin{array}{l}\text { Ostrácodos, detritos, algas, diatomeas, } \\
\text { quironómidos, cladóceros y otros } \\
\text { invertebrados. }\end{array}$ & $\begin{array}{l}\text { Observación personal } \\
\text { Baker y McLachlan (1979) } \\
\text { Henriques-Oliveira et al. (2003) }\end{array}$ \\
\hline Chelifera & $\begin{array}{l}\text { Larvas y pupas de quironómidos y } \\
\text { simúlidos Macroinvertebrados acuáticos. }\end{array}$ & $\begin{array}{l}\text { Wagner et al. (2011) } \\
\text { Tomanova et al. (2006) }\end{array}$ \\
\hline Hemerodromia & Microalgas & Granados (2013) \\
\hline Ferrisia, Physella Planorbis & Partículas del detrito, algas y bacterias & Cummins et al. (2005) \\
\hline
\end{tabular}

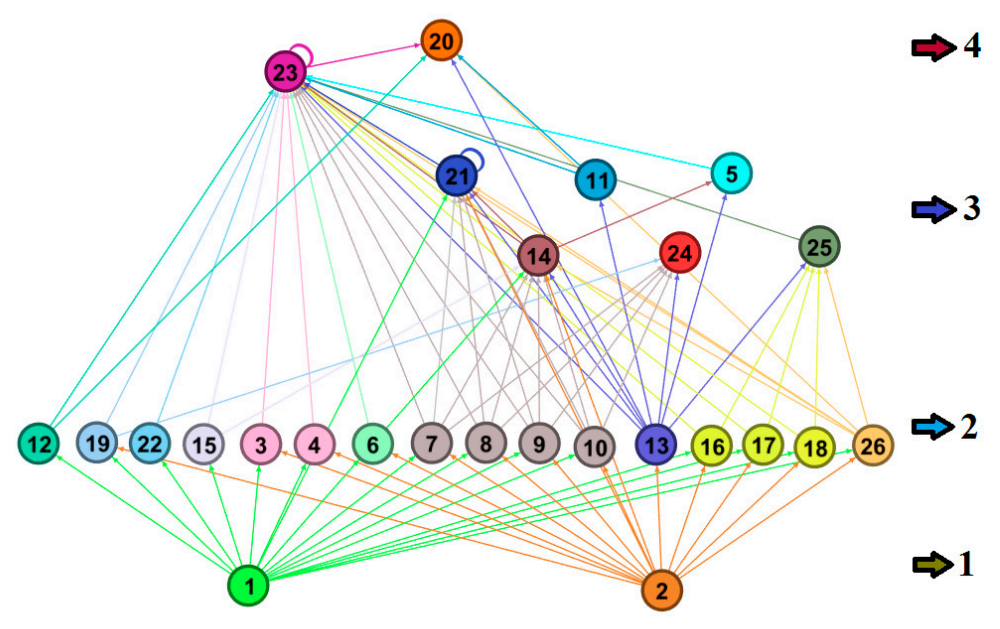

Figura 2. Red Trófica de los invertebrados acuáticos asociados a E. densa. 1) Perifiton 2) Detritus (3, 4 Trofoespecies de ácaros) 3) Hydrozetes cf. dimorphus 4) Trimalaconothrus cf. novus 5) Macrocyclops albidus 6) Harpacticoida (7, 8, 9, 10 Trofoespecies de cladóceros) 7) Eurycercus norandinus 8) Chydoridae (Grabtoleberis sp. y Alonella sp.) 9) Macrothrix 10) Simocephalus sp. 11) Chelifera sp. 12) Hemerodromia sp. 13) Dicrotendipes sp. 14) Ablabesmyia sp. 15) Cypridopsis vidua (16, 17, 18 Trofoespecies de gasterópodos) 16) Ferrisia sp. 17) Planorbis sp. 18) Physella sp. 19) Hyalella sp. 20) Gyrinus sp. 21) Corixidae 22) Oxyethira 23) Odonata (Ischnua hastata. y Coryphaesna sp.) 24) Girardia sp. 25) Helobdella sp. y 26) Nais sp. 


\section{DISCUSIÓN}

La red trófica generada en este estudio presenta cuatro niveles tróficos, característica común en redes tróficas acuáticas y terrestres las cuales cuentan con una cantidad de atributos constantes, que incluyen un pequeño número de niveles tróficos, usualmente tres o cuatro (Pimm y Kitching, 1987).

Los resultados de las fracciones de cada trofoespecie son similares a los obtenidos por Briand y Cohen (1984) y Warren (1989), en donde la fraccion de especies intermedias es mayor que las tope y las basales; caracteristica que se registra como constante para diferentes redes troficas independientemente del número de especies y de los hábitats estudiados (Pimm, 2002); sin embargo, estas características pueden variar entre tramas dependiendo del nivel de agrupamiento que utilice el investigador. Según Andrade-López (2012), las especies presentan un grado de sensibilidad al nivel de agregacion, para especies tope y basales es alto y para intermedias bajo.

En este caso, el modelo de red trófica cuenta con dos trofoespecies basales independientes que sustentan toda la comunidad, pero ambas dependen de un solo organismo que es E. densa: sustrato del perifiton (Giorgi y Feijoo, 2010) y fuente principal del detrito (Frauendorf et al., 2013), lo que hace a esta red trófica frágil en términos de robustez, ya que al afectarse esta planta, se altera toda la comunidad. La Robustez hace alusión al número de especies basales que es necesario retirar para que desaparezca el $50 \%$ de la comunidad (Dunne et al., 2002).

Sin embargo, esta red trófica se fortalece por la presencia de 10 trofoespecies consumidoras primarias que incluyen 16 taxones, lo que genera alta redundancia ecológica en términos de relaciones funcionales, garantizando el flujo de energía hacia los niveles superiores y la circulación de nutrientes (Covich et al., 1999). Esto lo refuerza el hecho de que sólo hay una trofoespecie (Dicrotendipes sp.) con ocho salidas, y una trofoespecie (Nais sp.) con cinco salidas, que transfieren energía a los consumidores superiores. Las otras ocho trofoespecies tienen en promedio dos salidas. Un caso similar es descrito por Montoya y Sole (2003), para una red trófica con una especie basal (Enteromorpha sp.), 16 especies intermedias y una de tope (Phalacrocorax carbo).

Dentro de las trofoespecies intermedias se reportan dos taxones con comportamiento omnívoro, es decir aquellos que se alimentan de más de un nivel trófico. Según Sánchez-Carmona et al. (2012), constituyen un factor fuertemente estabilizador en las redes alimentarias, en este caso, el hemíptero: Corixidae, puede consumir diferentes recursos que incluyen detritus, perifiton y pequeños insectos de cuerpo blando (Martin, 1969; Tomanova y Usseglio-Polatera, 2007), y el quironómido Ablabesyia sp., en algunos estudios considerado como depredador (Blanco-Belmonte, 1990; Rodríguez-Barrios et al., 2011; Rivera et al., 2013) y en otros como colector-recolector (Chará-Serna et al., 2010). Este tipo de consideraciones muestra la alta plasticidad que exhiben algunos taxones neotropicales en sus hábitos alimenticios, que muestran tendencias a la omnivoría, característica que les facilita adaptarse a las condiciones fluctuantes del trópico (Tomanova et al., 2006; Chará-Serna et al., 2010; Guzmán-Soto y Tamaris-Turizo, 2014).

Respecto a los depredadores se registran ocho trofoespecies que incluyen desde copépodos (Macrocyclops albidus), hasta coleópteros (Gyrinus sp.), los cuales ocupan diferentes niveles tróficos, desde trofoespecies intermedias (omnívoras, caníbales) y hasta de tope. Se resalta la presencia de los odonatos Ischnura hastata y Coryphaeschna sp., los cuales constituyen especies clave como reguladores de la comunidad con 22 entradas de energía, que incluyen el canibalismo como retroalimentación positiva, lo que constituye una importante función en la dinámica poblacional de otros invertebrados acuáticos y afectan directamente sus densidades e indirectamente modifican su distribución espacial (Springer et al., 2010).

Dentro de los atributos de la red trófica, la conectancia o número potencial de enlaces (May, 1973; Pimm, 1982) fue de 0,13 , valor que es considerado una conectividad media en el sistema. Esta variable tiende a disminuir cuando la meiofauna es incluida, sugiriendo una compensación entre la integridad y la resolución taxonómica (Thompson et al., 2012) y cuando la riqueza de especies es alta (Murtaugh y Kollath, 1997; Montoya y Sole, 2003). Sin embargo, cuando el detritus es incluido como trofoespecie basal en las redes tróficas, la conectancia tiende a aumentar (Pimm et al., 1991), este valor tambien puede ser atribuido a la presencia de $E$. densa, ya que, provee un sustrato estable y estructuralmente más complejo que el sedimento y el plancton (Motta y Uieda, 2005).

Con relación a la densidad de conexiones, para esta red se obtuvo 2,95 , valor que mide el número 
de vínculos tróficos por trofoespecies o especies taxonómicas (Pimm et al., 1991). Inicialmente esta característica de las redes, se planteó como un valor constante y cercano a 1,86 (Briand y Cohen, 1984). Sin embargo, diversas investigaciones como las de Martínez (1991; 1992), Dunne et al. (2004) muestran que este, no es un atributo constante, por el contrario, es muy variable y depende directamente de los taxones involucrados, convirtiendose en una propiedad poco robusta para la comparación entre tramas de diferentes tamaños y resoluciones.

\section{CONCLUSIONES}

Se destaca la construcción de un modelo de red trófica que permite diferenciar los patrones fundamentales en la organización trófica de invertebrados en la zona litoral de un lago de alta montaña tropical, donde se evidencia la importancia de los invertebrados acuáticos (incluyendo la meiofauna) en el flujo de energía entre trofoespecies basales y superiores. Estos organismos muestran variadas estrategias de alimentación, resaltando la inclusión del detritus como trofoespecie esencial. La red trófica generada en este estudio se encuentra conformada por dos trofoespecies basales, 16 intermedias y dos de tope; este hecho muestra baja robustez y alta redundancia ecológica en la comunidad de invertebrados acuáticos. Por último sobresale el rol de E. densa como una planta acuática estructuradora de la comunidad, por la generación de detritus y de sustrato para perifiton y las dos trofoespecies basales que soportan la red.

\section{AGRADECIMIENTOS}

Agradecemos a la Dirección de Investigaciones (DIN) de la Universidad Pedagógica y Tecnológica de Colombia por el apoyo a través de la convocatoria 014-2014. A los especialistas: Santiago Gaviria del Konsulent fuer Gewaesseroekologie und Systematische BiologieAustria, Rodulfo Ospina de la Universidad Nacional de Colombia-Sede Bogotá, Rodolfo Novelo del Instituto de Ecología INECOL y a Margarita Ojeda del Instituto de Biología UNAM, por su ayuda en la confirmación taxonómica de algunos organismo. A los evaluadores y editores por sus valiosos comentarios al manuscrito.

\section{BIBLIOGRAFÍA}

Andrade-López, J. 2012. Estructura de las tramas tróficas en ríos neotropicales: Consideraciones metodológicas en el análisis de atributos y procesos. Editorial Académica Española.
Bastian, M., Heymann, S. y Jacomy, M. 2009. Gephi: an open source software for exploring and manipulating networks. International AAAI Conference on Weblogs and Social Media 361-362.

Berghe, V. y Bergmans, M. 1981. Differential Food Preferences in Three Co-Occurring Species of Tisbe (Copepoda, Harpacticoida). Marine Ecology - Progress Series 4: 273-219.

Blanco-Belmonte, L. 1990. Estudio de las comunidades de invertebrados asociados a las macrófitas acuáticas de tres lagunas de inundación de la sección baja del Rio Orinoco, Venezuela. Sociedad de Ciencias Naturales La Salle.

Briand, F. y Cohen, J. 1984. Community food webs have scale-invariant structure. Nature 307(5948): 264-267.

Brinkhurst, R. y Marchese, M. 1991. Guía para la identificación de oligoquetos acuáticos continentales de Sud y Centro América. Colección Cimax. Segunda edición. A.C. Litoral, Editorial Santo Tomé, Argentina.

Brusca, R. y Brusca, G. 2003. Invertebrates. Second Edition. Sinauer Associates, Inc., Massachusetts.

Caraballo, P. 2009. Uso de isótopos estables de carbono y nitrógeno para estudios de ecología acuática. Boletín Cientifico CIOH 27: 176-178.

Caraballo, P., Forsberg, B. y Leite, R. 2014. Seasonal Variation in the Distribution and Isotopic Composition of Phytoplankton in an Amazon Floodplain Lake, Brazil. Acta Biológica Colombiana 19(2): 291-304.

Cardozo, A., Bermúdez, A., Aranguren, N. y Duque, S. 2005. Algas planctónicas del lago de Tota: listado taxonómico actualizado. Ciencia en Desarrollo 1: 81-88.

Carrillo, Y., Guarin, A. y Guillot, G. 2006. Biomass distribution, growth and decay of Egeria densa in a tropical high-mountain reservoir (NEUSA, Colombia). Aquatic Botany 85: 7-15. Doi: 10.1016/j.aquabot.2006.01.006.

Chará-Serna, A., Chará, J., Zúñiga, M.D., Pedraza, G. y Giraldo, L. 2010. Clasificación trófica de insectos acuáticos en ocho quebradas protegidas de la ecorregión cafetera colombiana. Universitas Scientiarum 15(1): 27-36.

Cordero, R., Ruiz, E. y Vargas, E. 2005. Determinación espacio-temporal de la concentración de fósforo en el lago de Tota. Revista Colombiana de Química 34(2): 211-218.

Corpoboyaca. 2015. Flujo de Nutrientes (C, N y P) en el Lago de Tota: Análisis de fuentes y biocaptación. Informe Final- Convenio, Boyacá, Aquitania: CorpoBoyacáUniversidad Pedagógica y Tecnológica de Colombia. 
Covich, A., Palmer, M. y Crowl, T. 1999. The role of benthic invertebrate species in freshwater ecosystems. BioScience 49(2): 119-127.

Croteau, M.N., Luoma, S.N. y Stewart, A.R. 2005. Trophic transfer of metals along freshwater food webs: evidence of cadmium biomagnification in nature. Limnology and Oceanography 50(5): 1511-1519. Doi: 10.4319/ lo.2005.50.5.1511.

Cummins, K., Merritt, R. y Andrade, P. 2005. The use of invertebrate functional groups to characterize ecosystem attributes in selected streams and rivers in south Brazil. Studies on Neotropical Fauna and Environment 40(1): 6989. Doi: 10.1080/01650520400025720.

De Ruiter, P., Wolters, V., Moore, J. y Winemiller, K. 2005. Food Web Ecology: Playing Jenga and Beyond. Science 309(5731): 68-71.

Domínguez, E. y Fernández, R. 2009. Macroinvertebrados Bentónicos Sudamericanos. Sistematica y Biología. Fundación Miguel Lilio, Tucuman, Argentina.

Dumont, H. y Negrea, S. 2002. Guides to the Identification of the Microinvertebrates of the Continental Waters of the World. Backhuys Publishers, Leiden.

Dunne, J.A. 2009. The Network Structure of Food Webs. Workshop on Theoretical Ecology and Global Change, En: Pascual, M. y Dunne, J.A., Editores. Ecological Networks: Linking Structure to Dynamics in Food Webs. Oxford University Press, New York.

Dunne, J.A. y Williams, R.J. 2009. Cascading extinctions and community collapse in model food webs. Philosophical transactions of the royal society 364: 1711-1723. Doi: 10.1098/rstb.2008.0219.

Dunne, J.A., Williams, R.J. y Martinez, N.D. 2004. Network structure and robutness of marine food webs. Marine Ecology Progress Series 273: 291-302.

Dunne, J.A., Williams, R.J. y Martinez, N.D. 2002. Network structure and biodiversity loss in food webs: robustness increases with connectance. Ecology letters 5(4): 558-567.

Elmoor-Loureiro, L. 1997. Manual de identificação de Cladóceros Límnicos do Brasil. Editora Universa, Brasilia.

Epler, J. 1992. Identification Manual for the Larval Chironomidae (Diptera) of Florida. Florida Department of Environmental Regulation, Orlando.
Esteves, F. 2011. Fundamentos de Limnología. $3^{\circ}$ edición. Editora Interciência, Rio de Janeiro.

FlöBner, D. 2000. Die Haplopoda und Cladocera (ohne Bosminidae). Backhuys Publishers, Leiden.

Frauendorf, T., Colón-Gaud, C., Whiles, M., Barnum, T., Lips, K., Pringle, C. y Kilham, S. 2013. Energy flow and the trophic basis of macroinvertebrate and amphibian production in a neotropical stream food web. Freshwater Biology 58(7): 1340-1352. Doi: 10.1111/fwb.12131.

Fryer, G. 1968. Evolution and Adaptive Radiation in the Chydoridae (Crustacea: Cladocera): A Study in Comparative Functional Morphology and Ecology. Editado por The Royal Society. Biological Sciences 254(795): 221-385.Doi: 10.1098/ rstb.1968.0017.

Gaviria, S. y Aranguren, N. 2003. Guía de laboratorio para identificación de Cladóceros (Anomopoda y Ctenopoda) y Copépodos (Calanoida y Cyclopoida). Univeridad Pedagógica y Tecnológica de Colombia, Tunja.

Giacomini, H. 2007. Os mecanismos de coexistência de espécies como vistos pela teoria ecológica. Oecologia Brasiliensis 11(4): 521-543.

Giacomini, H. y Petrere, M. 2010. A estrutura de teias tróficas. Boletim da Sociedade Brasileira de Limnologia 38(1): 1-33.

Giorgi, A. y Feijoó, C. 2010. Variación temporal de la biomasa del perifiton de Egeria densa Planch en un arroyo pampeano. Limnética 29: 269-278.

Gil, L. y Aranguren, N. 2013. Estimación de la biomasa de la comunidad de macroinvertebrados asociados a Egeria densa en el Lago de Tota - Boyacá. Boyacá, Corpoboyacá Universidad Pedagógica y Tecnología de Colombia, Tunja.

Gil-Padilla, N., Pedroza-Ramos, A. y Aranguren-Riaño, N. 2016. Valoración ambiental del litoral del lago de Tota basado en estructura y función de macroinvertebrados. Cultura Científica 14: 16-25.

Granados, C. 2013. Análisis de la dieta de los macroinvertebrados bentónicos en un gradiente altitudinal de la cuenca del Rio Gaira (Sierra-Nevada de Santa MartaColombia). Tesis de maestría, Universidad del Zulia, Maracaibo, Venezuela.

Guisande, C., Barreiro, A., Maneiro, I., Riveiro, I., Vergara, A. y Vaamonde, A. 2006. Tratamiento de Datos. Ediciones Díaz de Santos, España. 
Guzmán-Soto, C. y Tamaris-Turizo, C. 2014. Hábitos alimentarios de individuos inmaduros de Ephemeroptera, Plecoptera y Trichoptera en la parte media de un río tropical de montaña. Revista de Biología Tropical 62: 169-178.

Henriques-Oliveira, H., Nessimian, J. y Dorvillé, L. 2003. Feeding Habits of Chironomid Larvae (Insecta: Diptera) from a stream in the Floresta Tijuca, Rio de Janeiro, Brazil. Brazilian Journal of Biology 63(2): 269-281.

Hernani, A. y Ramírez, J. 2002. Aspectos morfométricos y teóricos de un embalse tropical de alta montaña: represa La Fe, El Retiro, Colombia. Revista de la Academia Colombiana de Ciencias Exactas, Físicas y Naturales 26(101): 511-518.

Howard-Williams, C. y Lenton, G. 1975. The role of the littoral zone in the functioning of a shallow tropical lake ecosystem. Freshwater Biology 5: 445-459. Doi: 10.1111/ j.1365-2427.1975.tb00147.

Ironmonger, J.W. y Young, J.O. 1980. A laboratory study of the food of three species of leeches occurring in British lakes. Hydrobiologia 68: 209-215.Doi: 10.1007/BF00018828.

Keiper, J.B., Casamatta, A. y Foote, A. 1998. Use of algal monocultures by larvae of Hydroptila waubesiana and Oxyethira pallida (Trichoptera: Hydoptilidae). Hydrobiologia 380: 87-91. Doi: 10.1023/A: 1003468432655.

Klecka, J. 2014. The role of a water bug, Sigara striata, in freshwater food webs. PeerJ 2:1-16. Doi: 10.7717/peerj.389.

Limén, H., Colin, D., Overdijk, V. y MacIsaac, H. 2005. Food Partitioning between the Amphipods Echinogammarus ischnus, Gammarus fasciatus, and Hyalella azteca as Revealed by Stable Isotopes. Journal of Great Lakes Research 31(1): 97-104.

Margalef, R. 1983. Limnología. Ediciones Omega, Barcelona.

Marten, G. 1990. Elimination of Aedes albopictus from tire piles by introducing Macrocyclops albidus (COPEPODA, CYCLOPIDAE). Journal of the american mosquito control association 6(4): 689-693.

Martin, N. 1969. The food feeding mechanism and ecology of the Corixidae (Hemiptera-Heteroptera), with special reference to Leicestershire. Doctoral Thesis, University of Leicester, Leicester, England.

Martínez, N. 1991. Artifacts or attributes? Effects of resolution on the little rock lake food web. Ecological Monographs 61(4): 367-392.
Martínez, N. 1992. Constant connectance in community food webs. The American Naturalist 139(6): 1208-1218.

May, R. 1973. Stabilty and complexity in Model Ecosystems. Princeton University Press, Oxford.

McCafferty, P. y Provonsha, A. 1981. Aquatic Entomology. Science Books International, Boston.

Merrit, M., Cummins, K. y Berg, M. 2008. An introduction to the aquatic insects of North America. Kendall Hunt Publishing Company, Dubuque.

Mojica, A. y Guerrero, J. 2013. Evaluación del movimiento de plaguicidas hacia la cuenca del Lago de Tota, Colombia. Revista Colombiana de Química 42(2): 1-27.

Montoya, J. y Sole, R. 2003. Topological properties of food webs: from real data to community assembly models. Oikos 102(3): 614-622. Doi: 10.1034/j.16000706.2003.12031.x.

Moore, J., Berlow, E., Coleman, D., De Ruiter, P., Dong, Q., Hastings, A., Collings, N., McCann, K., Melville, K., Morin, P., Nadelhoffer, K., Rosemond, A., Post, D., Sabo, J., Scow, K., Vanni, M., Wall, D. 2004. Detritus, trophic dynamics and biodiversity. Ecology Letters 7: 584-600. Doi: 10.1111/j.1461-0248.2004.00606.x.

Motta, R. y Uieda, V. 2005. Food web structure in a tropical stream ecosystem. Austral Ecology 30(1): 58-73. Doi:10.1111/j.1442-9993.2005.01424.x.

Murtaugh, P. y Kollath, J. 1997. Variation of trophic fractions and connectance in food webs. Ecology 78(5): 1382-1387. Doi: $10.2307 / 2266133$.

Nash, J.E. y Sutcliffe, J.V. 1970. River flow forecasting through conceptual models part I- A discussion of principles. Journal of Hydrology 10(3): 282-290.

Norton, R., Williams, D., Palmer, I. y Hogg, S. 1988. Biology of the oribatid mite Mucronothrus nasalis (Acari: Oribatida: Trhypochthoniidae) from a samll colwater springbrook in eastern Canada. Canadian Journal of Zoology 66(3): 622629. Doi: 10.1139/z88-093.

Novelo, R. y Gonzalez, E. 1991. Odonata de la Reserva de la Biosfera la Michilia, Durango, Mexico. Parte II. Nayades. Folia Entomológica Mexicana 81: 107-164.

Oscoz, J., Galicia, D. y Miranda R. 2011. Identification Guide of Freswater Macroinvertebrates of Spain. Springer, Navarra. 
Pelicice, F., Agostinho, A. y Thomaz, S. 2005. Fish assemblages associated with Egeria in a tropical reservoir: investigating the effects of plants biomass and diel period. Acta Oecologica 27: 9-16. Doi: 10.1016/j.actao.2004.08.004.

Pickavance, J. 1971. The diet of the immigrant planarian Duegesia tigrina (Grirard). Journal of Animal Ecology 40(3): 637-650. Doi: 10.2307/3442.

Pimm, S. 2002. Food Webs. The University of Chicago Press, London.

Pimm, S., Lawton, J. y Cohen, J. 1991. Food web patterns and their consequences. Nature 350: 669-674.

Pimm, S. y Kitching, R. 1987. The determinants of food chain lenghts. Oikos 50(3): 302-307. Doi: 10.2307/3565490.

Pimm, S. 1982. Food Webs. Chapman and Hall, New York.

Pennak, R. 1989. Fresh-Water invertebrates of the United States. Protozoa to Mollusca. Third Edition. WileyInterscience Publication, New York.

Pritchard, G. 1964. The Prey of Dragonfly Larvae (Odonata; Anisoptera) in Ponds in Northern Alberta. Canadian Journal of Zoology 42:785-800.

Ramírez, A. y Gutiérrez-Fonseca, P. 2014. Functional feeding groups of aquatic insect families in Latin America: a critical analysis and review of existig literature. Revista de Biología Tropical 62: 155-167.

Ricaurte, P. 2005. Plan de ordenación y manejo de la Cuenca de Tota - Problemática Ambiental. Corporación Autónoma de Boyacá.

Rico-Sánchez, A.E., Rodríguez-Romero, A.J., López-López, E. y Sedeño-Díaz, J.E. 2014. Spatial and temporal variation patterns in aquatic macroinvertebrates of Tecocomulco Lake, Hidalgo (México). International Journal of Tropical Biology and Conservation 62: 81-96.

Rivera, J., Pinilla, G. y Camacho, D. 2013. Grupos Tróficos de Macroinvertebrados Acuáticos en un Humedal Urbano Andino de Colombia. Acta Biológica Colombiana 18(2): 279-292.

Roca, J., Baltanas, A. y Uiblein, F. 1993. Adaptive responses in Cypridopsis vidua (Crustacea: Ostracoda) to food and shelter offered by a macrophyte (Chara fragilis). Hydrobiologia 262:127-131.Doi: 10.1007/BF00007513.

Rodríguez-Barrios, J., Ospina-Tórres, R. y TurizoCorrea, R. 2011. Grupos Funcionales alimentarios de macroinvertebrados acuáticos en el río Gaira, Colombia. Revista de Biologia Tropical 59(4): 1537-1552.
Roldán, G. 1996. Guía Para el Estudio de los Macroinvertebrados Acuáticos del Departamento de Antioquia. Fondo para la Protección del Medio Ambiente, Bogotá.

Ruiz-Moreno, J., Ospina-Torres, R., y Wolfgang, R. 2000. Guía para la Identificación Genérica de Larvas de Quironómidos (Diptera: Chironomidae) de la Sabana de Bogotá. II. Subfamilia Chironominae. Caldasia 22(1): 15-33.

Ruíz, E. 2002. Métodos para el estudio de las características Físico-Químicas del agua. En: Rueda, G., Editor. Manual de Métodos en Limnología, Asociación Colombiana de Limnología, Bogotá.

Salgado-Negret, B. 2015. La ecología funcional como aproximación al estudio, manejo y conservación de la biodiversidad: protocolos y aplicaciones. Instituto de Investigación de Recursos Biológicos Alexander von Humboldt, Bogotá.

Sánchez-Carmona, R., Rodríguez-Ruiz, A., EncinaEncina, L., Rodríguez-Sánchez, M. y Granado-Lorencio, C. 2012. Consequences of species grouping for food web structure. Applied ecology and environmental research 10(3): 333-347.

Schindler, D. y Scheuerell, M. 2002. Habitat coupling in lake ecosystems. Oikos 98(2): 177-189. Doi: 10.1034/j.16000706.2002.980201.x.

Smith, J. y Kaster, M. 1986. Feeding habits and dietary overlap of Naididae (Oligochaeta) from a bog stream. Hydrobiologia 137: 193-201.Doi: 10.1007/BF00004233

Springer, M., Ramírez, A. y Hanson, P. 2010. Macroinvertebrados de Agua Dulce de Costa Rica I. Revista Biologia Tropical 58(Suppl4).

Suárez, S., Rodríguez, J., Menéndez, Z., Montada, D., García, I. y Marquetti. Fernández, M. 2005. Macrocyclops albius (Copepoda: Cyclopidae): una nueva alternativa para el control de larvas de mosquitos en Cuba. Revista Cubana de Medicina Tropical 57(3) NA.

Thompson, R., Dunne, J. y Woodward, G. 2012. Freshwater food webs: towards a more fundamental understanding of biodiversity and community dynamics. Freshwater Biology 57(7): 1329-1341. Doi: 10.1111/j.1365-2427.2012.02808.x.

Thorp, J. y Covich, A. 2010. Ecology and Classification of North American Freshwater Invertebrates. Academic Press, San Diego. 
Tomanova, S., Goitia, E. y Helesic, J. 2006. Thophic levels and functional feeding groups of macroinvertebrates in neotropical streams. Hidrobiologia 556(1): 251-264. Doi: $10.1007 /$ s10750-005-1255-5.

Tomanova, S. y Usseglio-Polatera, P. 2007. Patterns of benthic community traits in neotropical streams: relationship to mesoscale spatial variability. Hydrobiologia 170(3): 243-255. Doi: 10.1127/1863-9135/2007/0170-0243.

Thomaz, S. y Cunha, E. 2010. The role of macrophytes in habitat structuring in aquatic ecosystems: methods of measurement, causes and consequences on animal assemblages, composition and biodiversity. Acta Limnologica Brasiliensia 22(2): 218-236. Doi: 10.4322/ actalb.02202011.

Vadeboncoeur, Y., Vander, J. y Lodge, D. 2002. Putting the lake back together: Reintegrating benthic pathways into lake food web models. BioScience 52(1): 44-54. Doi:10.1641/0006-3568(2002)052[0044:PTLBTR]2.0.CO;2

Wagner, R., Marxsen, J., Zwick, P. y Cox, E. 2011. Central European Stream Ecosystems. Wiley-Blackwell, Weinheim.
Walsh, G.C., Dalto, Y.M., Mattioli, F.M., Carruthers, R.I. y Anderson, L.W. 2013. Biology and ecology of Brazilian elodea (Egeria densa) and its specific herbivore, Hydrellia sp., in Argentina. BioControl 58(1): 133-147.

Warren, P. 1989. Spatial and temporal variation in the structure of a freshwater food web. Oikos 55(3): 299-311.

Wetzel, R. y Likens, G. 2000. Limnological Analyses, Third Edition. Springer-Verlag, New York.

Wetzel, R. y Allen, H. 1972. Functions and interactions of dissolved organic matter and the littoral zone in lake metabolism and eutrophication. En: Kajak, Z. y HillbrichtIlkowska, A., Editores. Productivity Problems of Freshwaters. Polish Scientific Publication, Warszawa-Krakow.

Yarrow, M., Marin, V.H., Finlayson, M., Tironi, A., Delgado, L.E. y Fischer, F. 2009. The ecology of Egeria densa Planchon (Liliopsida: Alismatales): A wetland ecosystem engineer. Revista Chilena de Historia Natural 82(2): 299-313. Doi: http://dx.doi.org/10.4067/S0716078X2009000200010.

Fecha de recepción: 16/05/2016 Fecha de aceptación: 06/09/2016

Para citar este artículo: Pedroza-Ramos, A., Caraballo, P. y Aranguren-Riaño, N. 2016. Estructura trófica de los invertebrados acuáticos asociados a Egeria densa (Planch. 1849) en el lago de Tota,

(Boyacá-Colombia). Revista Intropica Vol. 11: 21 - 34 